\title{
A rare cause of acute post renal failure: Retroperitoneal fibrosis
}

\author{
Kamil Gokhan Seker, Mithat Eksi, Yunus Colakoglu, Mustafa Gürkan Yenice, Fatih Gokhan Akbay, \\ Volkan Tugcu, Abdulmuttalip Simsek
}

Bakirkoy Sadi Konuk Research and Training Hospital, Department of Urology, Istanbul, Turkey.

\begin{abstract}
Summary Retroperitoneal fibrosis is an inflammatory process which may cause acute renal failure. In patients who admitted to emergency services with obstructive uropathy, retroperitoneal fibrosis should be considered in the differential diagnosis. We present our ten cases who admitted to emergency department with obstructive acute renal failure related to retroperitoneal fibrosis.
\end{abstract}

KEY WORDS: Retroperitoneal fibrosis; Postrenal acute renal failure; Obstructive uropathy.

Submitted 18 July 2017; Accepted 23 September 2017

\section{INTRODUCTION}

Retroperitoneal fibrosis (RF) is a clinical entity that causes inflammatory and fibrotic reactions in retroperitoneal organs and produces obstruction in ureters. It was first described by Albarran in 1905 and in 1948, the idiopathic form was described as Ormond's disease $(1,2)$. Recently, an international consensus has begun to use the term "IgG4-related disease (IgG4-RD)" which defines several organ-related disorders (3). The incidence is about $0.1-1.3 / 100.000$. RF generally seen over 50 years of age and 3 times more often in men $(4,5)$. Two-thirds of RF cases are idiopathic. Other causes include such as drugs, malignancies, infectious processes, radiotherapy, trauma and previous abdominal surgery (6).

RF may cause the symptoms of hydronephrosis and uremia resulting from the external pressure of the retroperitoneum and the deterioration of the ureteral passage. In this study, we aimed to present the diagnosis and treatment management of ten patients who were admitted to the emergency department (ED) with postrenal acute renal failure (ARF).

\section{Materials AND Methods}

We retrospectively analyzed the records of patients diagnosed with postrenal ARF caused by RF in our clinic between 2013 and 2016. The RF was diagnosed according to the computed tomography (CT) images taken at the ED. Biopsy was not performed due to absence of atypical appearance and no need of surgical intervention during follow-up. Urinary system decompression was made by placement of double J (DJ) stent or percutaneous nephrosto- my tube (PNT). Further medical treatments were consulted with nephrology department. Demographic data, physical examination findings, laboratory data, radiological examinations, treatments modalities and follow-up data were retrospectively analyzed.

Patients were followed up with 3-monthly laboratory tests and radiological observations. At the end of the one-year follow-up period, the patients were evaluated by comparing their initial and recent CT.

Statistical analysis was performed using SPSS (IBM Statistical Package for the Social Sciences, New York, USA) 17.0 program.

\section{RESULTS}

The mean age was $65.2 \pm 20.2$ years. Male/female ratio was $8 / 2$. The demographic data of the patients were shown in Table 1. The side pain was the most frequent presenting symptom. CT scan revealed increase in retroperitoneal soft tissue density at the periaortic and periureteral area in 8 patients with addition perinephritic area in 2 patients. Unilateral renal atrophy was observed in two patients as a result of fibrosis.

After the initial intervention, medical therapy was applied. Corticostreoids (CS) were used as a first line therapy for all patients. The antihypertensive treatment of the patient who admitted with beta-blocker toxicity was changed to another antihypertensive drug. For maintenance therapy, additionally to corticosteroids; seven patients were treated with azathioprine (AZA) and one

Table 1.

Demographic datas and serum creatinine levels during follow up.

\begin{tabular}{lc} 
No. total patients & 10 \\
\hline Male/female $(\mathrm{n})$ & $8 / 2$ \\
\hline Age mean \pm SD (year) & $65.2 \pm 20.2$ \\
\hline Idiopathic/secondary & $9 / 1$ \\
\hline Serum creatinine levels $(\mathrm{mg} / \mathrm{dl})$ & \\
Mean \pm SD & $5.7 \pm 3.5$ \\
Initial & $1.1 \pm 0.2$ \\
After urinary system decompression & $0.9 \pm 0.1$ \\
After medical therapy &
\end{tabular}


Table 2.

Demographic datas and serum creatinine levels during follow up.

\begin{tabular}{|lcccccc|}
\hline Patient No. & Gender & Symptom & Urinary system decompression & Medical therapy & Follow-up (month) & Status \\
\hline 1 & M & Side pain, weakness & PNT + D-J & CS + AZA & 42 & Full regression \\
\hline 2 & F & Hypotension & D-J & CS + TMX & 27 & Regression \\
\hline 3 & M & Bilateral side pain & D-J & CS + AZA & 12 & Stable \\
\hline 4 & M & Bilateral side pain & PNT + DJ & CS + AZA & 12 & Regression \\
\hline 5 & F & Side pain, weakness & PNT & CS + AZA & 18 & Regression \\
\hline 6 & M & Bilateral side pain & PNT + DJ & CS + AZA & 24 & Stable \\
\hline 7 & M & Bilateral side pain & PNT & CS & 18 & Regression \\
\hline 8 & M & Fever, weakness & PNT + DJ + AZA & 30 & Full regression \\
\hline 9 & M & Fever, side pain & PNT & CS + AZA & 12 & Stable \\
\hline 10 & M & Abdominal pain & PNT + DJ & CS & 18 & Regression \\
\hline
\end{tabular}

Figure 1.

A. Bilateral hydroureteronephrosis due to periureteral fibrosis.

B. Soft tissue density that extends from paraaortic area to caudal.

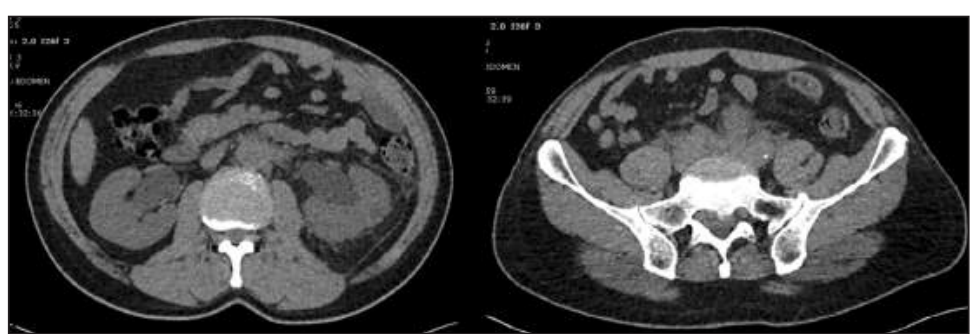

The mean initial serum creatinine level was 5.7 $\pm 3.5 \mathrm{mg} / \mathrm{dl}$. After the first intervention by decompressing urinary system, mean serum creatinine level regressed to $1.1 \pm 0.2 \mathrm{mg} / \mathrm{dl}$. At the end of medical therapy, the mean serum creatinine level was $0.9 \pm 0.1 \mathrm{mg} / \mathrm{dl}$.

Demographic data, presenting symptoms, laboratory and radiological findings, follow-up times and treatment data are summarized in Table 2.

Patient's radiological images were shown in Figures 1-5.

\section{Figure 2.}

A. Right $D J$ stent and left percutaneous nephrostomy tube.

B. Bilateral DJ stents.

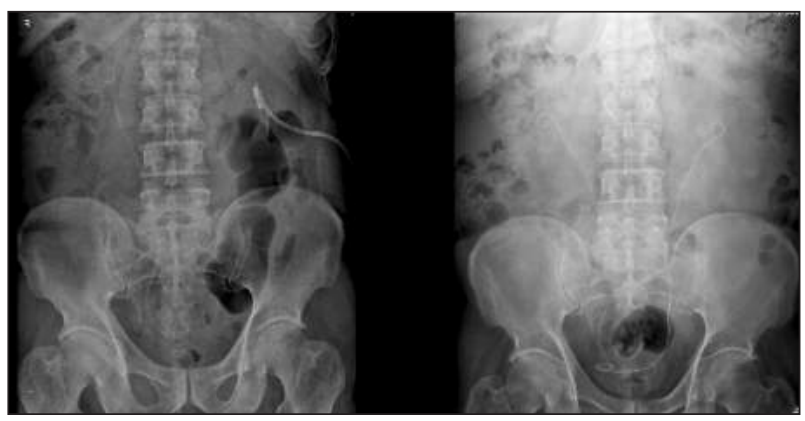

Figure 3.

Significant regression of the hydronephrosis and soft tissue density after steroid therapy.

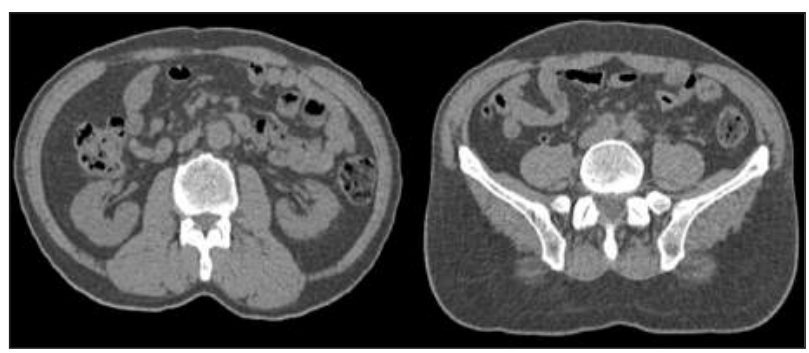

patient was treated with tamoxifen (TMX). Total regression and regression were observed in two and five patients respectively.

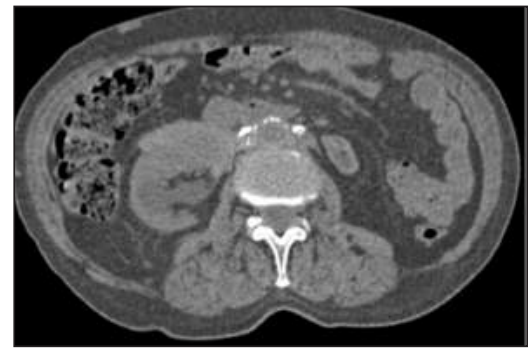

Figure 4.

Left atrophic kidney. Grade 2 hydronephrosis on the right kidney. Soft tissue enlargement and calcification extending around the large vessels and extending from the periureteral area to the pelvic region.

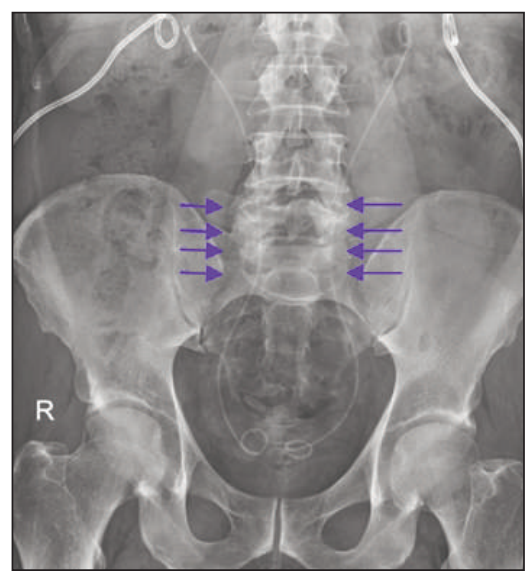

\section{Figure 5.}

Bilateral medial retraction due to periureteral fibrosis.

\section{Discussion}

Two-thirds of RF patients are idiopathic and the remaining are secondary to causes including infection, trauma, previous surgery and malignancy. In the literature, presence of 
retroperitoneal fibrosis following high doses of beta-blockers usage have been reported (7). In this study, we found that in 9 patients RF was idiopathic (90\%) and in one patient (10\%) it was related to drug overdose. Diagnosis of idiopathic RF is generally delayed due to atypical symptoms of the disease and patients may be diagnosed with postrenal ARF in ED. Urologically, the most frequent and most serious complication of the disease is ARF due to bilateral ureteral obstruction $(8,9)$. In two large studies in the literature, pain was the most common presenting symptom, and additionally undefined symptoms such as fatigue, weakness, myalgia were observed $(4,10)$. Renal failure due to bilateral ureteral obstruction is seen in $42-$ 95\% of the cases. Patients may also be referred to the urology clinic with scrotal edema, hydrocele or varicocele due to the involvement of the gonadal vessels (11). Erythrocyte sedimentation rate (ESR), C-Reactive protein (CRP), which are non-specific inflammation markers, are laboratory parameters used for diagnosis and follow-up $(12,13)$.

In our study, side pain and uremic symptoms due to bilateral ureteral involvement were observed in 8 patients.

The other two patients had different symptoms: one patient had hypotension and the other had fever and weakness. Initial inflammation markers were found to be high in all patients.

Non-contrast abdominal CT and magnetic resonance imaging (MRI) provide limited information in the diagnosis of the disease (14). In non-contrast CT, RF usually appears as an increase in soft tissue density, surrounding the abdominal aorta and iliac vessels, and usually surrounding the ureter and inferior vena cava (15). Positron emission tomography (PET-CT) provides more comprehensive clinical information as it includes whole-body metabolic studies. It shows active inflammation and post-treatment relapses, and reveals occult malignancies $(16,17)$. In our cases, we used non-contrast CT in consideration of the risk of contrast nephropathy due to the presence of ARF. During follow-up, if the creatinine level was in the normal range; contrast-enhanced MRI and CT examinations were performed.

In patients with radiologically diagnosed RF, which typically have mild symptoms and considered as a benign disease, medical treatment can be started without biopsy. Biopsy is usually preferred in cases of partial response or failure to the medical therapy (18). We did not perform biopsy because no progression was observed in any of our patients and CT had no atypical appearance.

The aim of the treatment is to relieve the fibro-inflammatory reaction, in order to reduce ureteral obstruction and prevent recurrence (19). After the urinary system decompression by PNT or DJ stent, there are two options as first line medical treatment (20). CS alone or CS with tamoxifen (TMX) can be used as first line therapy (21). The second step is the addition of drugs such as immunosuppressive azathioprine (AZA), mycophenol mofetil, rituximab, bortezomip, cyclophosphamide, cyclosporine in the course of treatment or relapse (22).

In the case of failure to respond to medical treatment, the treatment approach is adjuvant CS therapy after surgical obstruction is reduced by ureterolysis. Secondary RF is treated for the underlying cause (8). Urinary system decompression was achieved by DJ stent in two of our patients, by PNT in three patients and by both DJ stent and PNT placement in five other patients. In two of the 5 patients treated by both DJ and PNT, a DJ stent was inserted firstly but a PNT had to be inserted subsequently because of the lack of adequate radiological and clinical regression. After initial treatment, CS was initially administered with additionally AZA and TMX.

In a randomized controlled trial, after achieving remission with high-dose prednisolone as an induction therapy, patients were divided into 2 groups and followed up with CS (prednisone) or TMX mono therapy for 8 months. In this study, maintenance therapy with CS was associated with lower relapse rates, with a higher side effect profile than TMX group (21). Van der Bilt et al. reported that CSs were superior to TMX for induction therapy, but the rate of recurrence was lower in patients treated with TMX for the maintenance therapy (23).

Surgical treatment may be applied in case of non response to medical treatment or interruption of medical treatment due to side effects. Open, laparoscopic and robotic ureterolysis can be performed as a salvage procedure in case of failure with medical therapy (24). In our management, all patients were treated by consecutive three line therapy, initial decompression and achieving of improved renal function, induction with corticosteroids and maintenance therapy with CS or addition of AZA or TMX. With the success of this third line therapy, surgical treatment was not performed during the follow-up period.

Our study has some limitations such as the small sample size and as the retrospective design. In addition, there were no long-term follow-up results of patients.

\section{Conclusions}

Retroperitoneal fibrosis should be kept in mind in patients who admitted to the ED with hydroureteronephrosis and uremic symptoms. After the initial treatment with urinary system decompression, remission can be achieved with appropriate medical treatments.

\section{REFERENCES}

1. Albarran J. Retention renale par periureterite: Liberation externe uretere. Ass Fr Urol. 1905; 9:511.

2. Ormond JK. Bilateral ureteral obstruction due to development and compression by an inflammatory retroperitoneal process. J Urol. 1948; 59:1072.

3. Fairweather J, Jawad AS. Immunoglobulin G4 related retroperitoneal fibrosis: A new name for an old disease. Urology. 2013; 82:505 7 .

4. van Bommel EF, Jansen I, Hendriksz TR, Aarnoudse AL. Iodiopathic retroperitoneal fibrosis: prospective evaluation of incidence and clinicoradiologic presentation. Medicine (Baltimore) 2009; 88:193-201.

5. Nakada SY, Hsu THS. Retroperitoneal fibrosis. CampbellWalsh Urology, $10^{\text {th }}$ edition. Editorr: Wein AJ, Kavoussi LR, Novick AC, Partin AW, Peters CA. Philadelphia, ElsevierSaunders, 2012; 1165-1168

6. Palmisano A, Vaglio A. Chronic periaortitis: a fibro-inflammatory disorder. BestPract Res Clin Rheumatol 2009; 23:339-53.

7. Bullimore DW. Retroperitoneal fibrosis associated with metoprolol. Br Med J (Clin Res Ed). 1982; 284:664. 
8. Ross JC, Tinckler LF. Renal failure due to periureteric fibrosis. Br J Surg. 1958; 46:58.

9. Ha YJ, Jung SJ, Lee KH, Lee SW, et al. Retroperitoneal fibrosis in 27 Korean patients: single center experience. J Korean Med Sci. 2011; 26:985-90.

10. Scheel PJ Jr, Feeley N. Retroperitoneal fibrosis; the clinical, laboratory, and radiographic presentation. Medicine (Baltimore) 2009; 88:202-207.

11. Vaglio A, Buzio C. Chronic periaortitis: a spectrum of diseases. Curr Opin Rheumatol. 2005; 17:34-40.

12. Magrey MN, Husni ME, Kushner I, Calabrese LH. Do acutephase reactants predict response to glucocorticoid therapy in retroperitoneal fibrosis? Arthritis Rheum. 2009; 61:674-679.

13. An LM, Xu YF, Zhang ZL. Clinical features and prognostic analysis of retroperitoneal fibrosis in 32 patients (in Chinese). J Peking Univ (Health Sci). 2012; 44:265-9.

14. Horger M, Lamprecht HG, Bares R, et al. Systemic IgG4-related sclerosing disease: spectrum of imaging findings and differential diagnosis. AJR Am J Roentgenol 2012; 199:W276-W282.

15. George V, Tammisetti V, Surabhi V, Shanbhogue A. Chronic fibrosing conditions in abdominal imaging. Radiographics. 2013; 33:10531080.

16. Zhang J, Chen H, Ma Y, et al. Characterizing IgG4-related disease with 18F-FDG PET/CT: a prospective cohort study. Eur J Nucl Med Mol Imaging. 2014; 41:1624-1634.
17. Caiafa RO, Vinuesa AS, Izquierdo RS, et al. Retroperitoneal fibrosis: Role of imaging in diagnosis and follow up. Radiographics. 2013; 33:535-52.

18. Surcel C, Mirvald C, Pavelescu C, et al. Management of idiopathic retroperitoneal fibrosis from the urologist's perspective. Ther Adv Urol. 2015; 7:85-99.

19. Fry AC, Singh S, Gunda SS, et al. Successful use of steroids and ureteric stents in 24 patients with idiopathic retroperitoneal fibrosis: a retrospective study. Nephron Clin Pract. 2008; 108:213-220.

20. Urban ML, Palmisano A, Nicastro M, et al. Idiopathic and secondary forms of retroperitoneal fibrosis: a diagnostic approach. Rev Med Interne. 2015; 36:15-21.

21. Vaglio A, Palmisano A, Alberici F, et al. Prednisone versus tamoxifen in patients with idiopathic retroperitoneal fibrosis: an open-label randomised controlled trial. Lancet. 2011; 378:338-346.

22. Khosroshahi A, Wallace ZS, Crowe JL, et al. International consensus guidance statement on the management and treatment of IgG4-related disease. Arthritis Rheumatol. 2015; 67:1688-1699.

23. van der Bilt FE, Hendriksz TR, van der Meijden WA, et al. Outcome in patients with idiopathic retroperitoneal fibrosis treated with corticosteroid or tamoxifen monotherapy. Clin Kidney J. 2016; 9:184-91.

24. Omer A, Esen T Retroperitoneal fibroziste cerrahi tedavi: nelere dikkat edilmeli? Endoüroloji Bülteni 2013; 6:10-15.

\section{Correspondence}

Kamil Gokhan Seker, MD

Mithat Eksi, MD

Yunus Colakoglu, MD

Mustafa Gürkan Yenice, MD

Fatih Gokhan Akbay, MD

Volkan Tugcu, MD

Abdulmuttalip Simsek, MD

simsek76@yahoo.com

Bakirkoy Sadi Konuk Research and Training Hospital, Department of Urology,

Zuhuratbaba, Tevfik Saglam Cad. NO:11

Bakirkoy, Istanbul, Turkey 